\title{
Rare Earth Elements Geochemistry of Ceramics Excavated from Tongguanyao Site, China
}

\author{
Longjiang $\mathrm{Mao}^{1, \mathrm{a}}$, Jijing $\mathrm{Du}^{1}$, Duowen $\mathrm{Mo}^{2}$, Jinghong $\mathrm{Yang}^{3}$, Haibin $\mathrm{Gu}^{4}$
}

${ }^{1}$ School of Marine Sciences, Nanjing University of Information Science \& Technology,

Nanjing 210044, China;

${ }^{2}$ College of Urban and Environmental Sciences, Peking University, Beijing 100871, China

${ }^{3}$ State Key Laboratory for Mineral Deposits Research, Nanjing University, Nanjing 210093, China

${ }^{4}$ Hunan Provincial Institute of Cultural Relies and Archaeology, Changsha 410008, China

a Email: mlj1214@163.com

\begin{abstract}
Keywords: Rare earth elements; ceramics; ICP-MS; Tongguanyao site (TGY)
\end{abstract}
Abstract. Rare earth element (REE) compositions in ceramics from the Tongguanyao site (TGY) of China were determined by the inductively coupled plasma-mass spectrometry (ICP-MS) to compare their sources. Total REE concentrations of 14 samples range from 120.9 to $453 \mu \mathrm{g} \bullet \mathrm{g}-1$, with an average value of $228.5 \mu \mathrm{g} \bullet \mathrm{g}-1$. The inconformity of chondrite-normalized REE patterns suggests that pottery and porcelain have different material sources. The normalized REE patterns indicates similar trends between the raw materials and ceramics in the same period, as a higher concentration of REEs is presented in the ceramics, suggesting that REEs enriched during the production process of ceramics with high temperature. A different chondrite normalized REE pattern between the reticulated soil and ceramics implies that they had not a single source, probably mixed material sources.

\section{Introduction}

The concentrations and compositions of geochemical element, especially the trace and rare earth element (REE), of ceramics play a important role in the study of pottery and porcelain[1, 2]. The ceramics can be divided into several groups on the ground of similarities or dissimilarities using the trace and REE element data determined by chemical meters [2, 3]. The chemical data can be determined by utility of the inductively coupled plasma-mass spectrometry (ICP-MS) [4, 5] or X-ray fluorescence (XRF) [6, 7] or instrumental neutron activation analysis (INAA) [8, 9]. Because of high sensitivity, and multi-element capability, ICP-MS is more extensively used in archaeological study of ancient ceramics [2]. Therefore, trace elemental data, especially rare earth element (REE) determined by ICP-MS, can be used to trace their provenances from sediments and other materials [10-12] .

In this study, a total of 14 samples was determined and characterized as far their REE compositions. Among them, two of these samples came from reticulated soils (reticulated yellow soil and reticulated white soil), seven of these samples including ancient ceramic and raw materials came from excavations at Tongguanyao (TGY) site, and five samples got from modern ceramics and raw materials. The purpose aims to: (1) to compare REE compositions by using ICP-MS analyses data of ceramics and raw materials , (2) to demonstrate ICP-MS analytical method can be used to distinguish their sources between ceramics and raw materials.

\section{Materials and methods}

In 1957,1959, 1978, and 2011, a large number of shards of ancient Chinese ceramics were excavated at Tongguanyao site in Wangcheng county, by the Changsha Institute of Cultural Relics in Hunan province. In this study, 14 samples including ceramics and their materials were measured by ICP-MS.

Small portions were cut from the shards using a diamond saw, the water and toothbursh were used to wash the contaminated surface until exposing fresh surface. After drying in the oven for $24 \mathrm{~h}$ at $100{ }^{\circ} \mathrm{C} .14$ samples were powdered in an agate mortar till passing the 200 mesh sieve for chemical 
analysis. $50 \mathrm{mg}$ powdered sediments were digested in the high pressure digestion tanks for chemical analysis. The analytical procedures was as follow: (1) Add $4 \mathrm{ml}$ of concentrated nitric acid to the tanks and allowed to stand 12 hours, so that the gas nitric acid naturally run it over. (2) Add $2 \mathrm{ml}$ hydrofluoric acid and $1 \mathrm{ml}$ hydrogen peroxide to the samples, then replace it in the digestion tank and heat in the oven at $100^{\circ} \mathrm{C}$ and heated to $180{ }^{\circ} \mathrm{C}$ for 5 hours. (3) After digestion, stay cool completely before opening. Then adjust final volume prior to measurement [13]. The reagent blanks and duplicate samples were also analyzed to ensure the reproducibility and accuracy of the results. The precision was estimated to be less than $5 \%$ based on duplicate analysis of samples and standards.

\section{Results and discussion}

REEs concentrations in ceramics and raw materials. The total contents of REEs in the samples varies from 120.9 to $453 \mu \mathrm{g} \bullet \mathrm{g}-1$, with an average value of $228.5 \mu \mathrm{g} \bullet \mathrm{g}-1$. Highest content of total REEs of $453 \mu \mathrm{g} \bullet \mathrm{g}-1$ is present in sample TGY001. The relatively low contents of total REEs are present in samples TGY002-005 and 007, with the values of 149.7, 156.4, 135.3, 120.9 and 164.5 $\mu \mathrm{g} \bullet \mathrm{g}-1$, respectively.

The LREE/HREE ratios of 14 samples have an average value of 7.8, with a wide variation from 7.4 to 16.9. The values of Eu anomalies and $\mathrm{Ce}$ anomalies have an average values of 0.52 and 0.85 , with the variations from 0.28 to 0.61 and from 0.46 to 1.1 , respectively. This means that the samples show significant Eu negative anomalies and most of samples indicate slight Ce negative anomalies, in addition to sample TGY002 (significant negative anomalies) and samples TGY001 and TGY008 (slight positive anomalies). The values for other characteristics parameters of $(\mathrm{La} / \mathrm{Lu})_{\mathrm{N}},(\mathrm{La} / \mathrm{Yb})_{\mathrm{N}}$, $(\mathrm{La} / \mathrm{Sm})_{\mathrm{N}}$ and $(\mathrm{Gd} / \mathrm{Yb})_{\mathrm{N}}$ in samples averaged $14.35,12.79,4.03$ and 2.11 , with ranges range from 8.97 to 32.76 , from 8.09 to 29.79 , from 3.29 to 5.78 and from 1.57 to 3.87 , respectively.

Normalized-patterns of REEs in ceramics and raw materials. ICP-MS is an effective method for determining the chemical composition of fired clay and has great potential for chemically characterization prehistoric ceramic[14]. ICP-MS data, especially REE contents, have been used to normalize to the chondrite values[15]. This method can be extensively used for analyses of sediment sources.

In our study, chondrite normalized REE patterns in raw materials from TGY site, including raw materials from Ming Dynasty, Tang Dynasty, today, together with reticulated yellow soil and reticulated white soil, indicated almost identical LREE enrichment and HREE depletion with obvious negative Eu anomalies, except for the reticulated yellow soil and reticulated white soil (Fig.1a). The reticulated yellow soil shows more obvious LREE enrichment than other samples, whereas reticulated white soil indicates significant negative $\mathrm{Ce}$ and $\mathrm{Eu}$ anomalies. Through observation of the normalized REE patterns, it is easy to discern that the samples can be distinguished as two categories (Fig. 1b), the first category includes the samples TGY012, TGY013 and TGY014, which indicate almost identical distribution pattern (Fig. 1b). In contrast, the second category, the samples TGY010 and TGY011, indicates higher LREE/ HREE ratios, with the values of 10 and 12, respectively. The two categories of samples may imply at least two different material sources to analyzed ceramics. These results show that pottery (samples TGY010 and TGY011) and porcelain (samples TGY012, TGY013 and TGY014) have different material sources.

The chondrite normalized REE patterns in the raw materials and pottery from Tang Dynasty is shown in Fig.1c. The similar distribution patterns can be observed in samples TGY012 and TGY013. The uniform REE patterns may imply similar source in samples TGY012 and TGY013. On the other side, it also suggests that sample TGY013 was a failure in producing porcelain, possibly due to lack of skill. However, chondrite normalized REE patterns between the pottery sample TGY010 and porcelain samples TGY012 and TGY013 were obviously different, suggesting different raw material source. Similar trends between the raw material sample TGY005 and porcelain samples TGY012 and TGY013, as higher concentrations are present in porcelain samples TGY012 and TGY013, possibly caused by the high temperature during the firing process, which made the REE enrichment in porcelain. The similar trends in Ming and nowadays samples can be observed in Fig.1d and Fig.1e, 
respectively. Therefore, the REE content of ceramics was higher than that of the raw material in the same period, although they indicated similar chondrite normalized REE patterns.
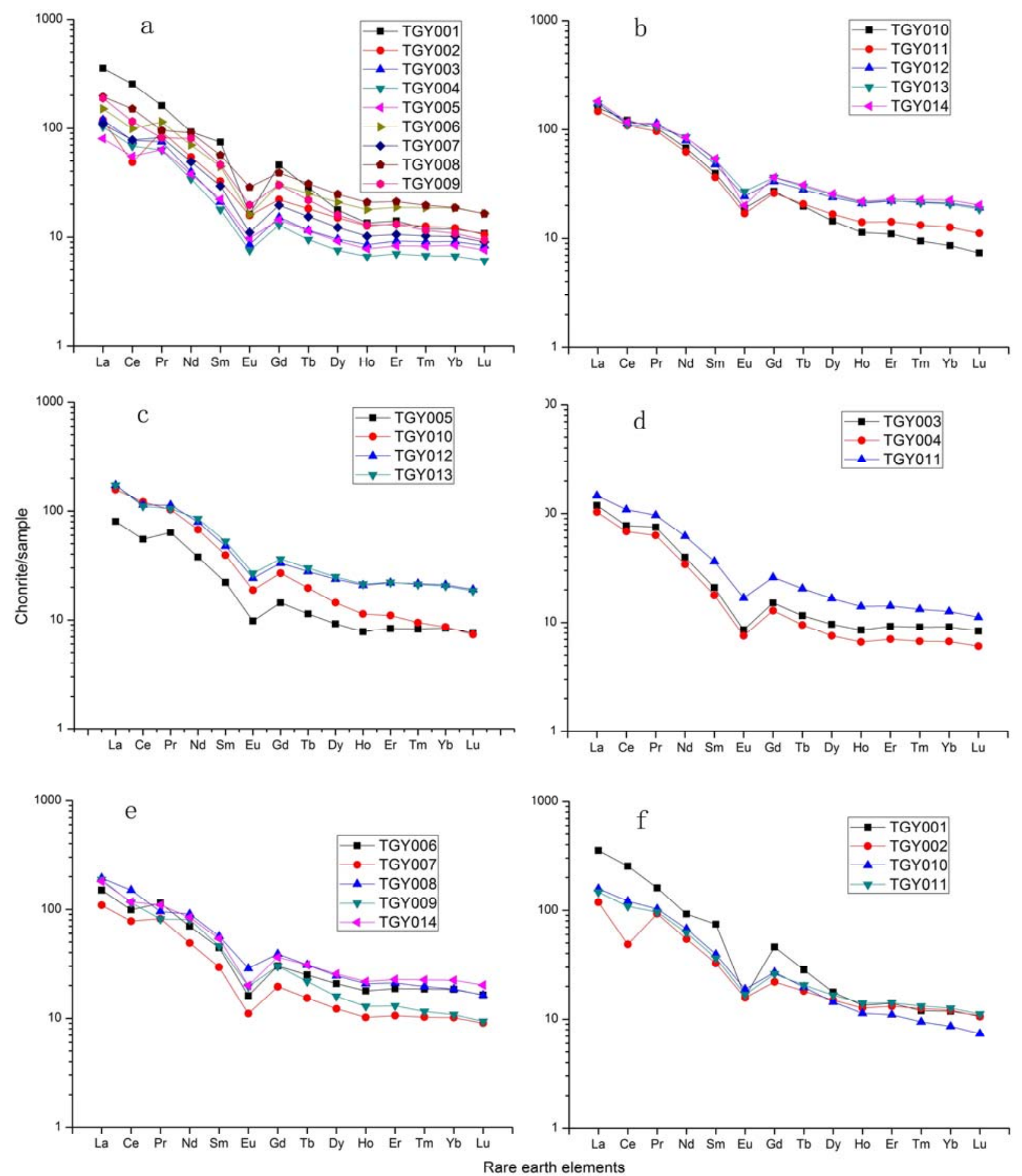

Fig.1 Chondrite-normalized REE patterns of ceramics and its materials from TGY site, China

In the Near East, most Tang sancai ceramics excavated are made of kaolinitic clay [16]. The middle and lower reaches of Yangtze River is characterized by widely distributed reticulated soil (reticulated yellow soil and reticulated white soil). High concentration of kaolinitic clay is presented in reticulated white soil [10]. However, there is a different chondrite normalized REE pattern between the reticulated white soil sample TGY002 and reticulated yellow soil TGY001 and other 
ceramic samples (Fig.1f), suggesting that reticulated white soil or reticulated yellow soil was not a single source, probably they were mixed with other material sources.

\section{Conclusions}

The ICP-MS, a well established method for geochemical elemental analysis, was used in this work to identify and quantify REEs in the ceramics and their raw materials. The REE composition and normalized REE patterns are also efficiently used for identification of the raw material sources.

\section{Acknowledgements}

This study was supported by the National Natural Science Foundation of China (No: 41271228) and National Science and Technology Support Program of China (No: 2013BAK08B02). Gratitude is expressed to Fu Q and Zhang XG for assisting in sampling.

\section{References}

[1] J.H. Burton and A.W. Simon: Amer. Antiq 58(1) (1993)45-59.

[2] M. Mallory-Greenough Leanne, D. Greenough John, Owen J Victor:J Archaeol SCI 25 (1998) 85-97.

[3] L. Li, G.X. Xie, S.L. Feng, X.Q. Feng, L.T. Yan, J.H. Zhu, Y.Q. Li, H.Y. Han:J Archaeol SCI 40 (2013)1449-1453.

[4] B.P. Li, A.Greig, J.X. Zhao, K.D. Collerson, K.S. Quan, Y.H. Meng, Z.L. Ma: J Archaeol SCI 32 (2005) 251-259.

[5] Eckert, Suzanne L. and James, William. D: J Archaeol SCI 38 (2011) 2155-2170.

[6] Speakman, Robert. J., Little Nicole C., Creel Darrell., Miller Myles R. and Iñañez Javier G: J Archaeol SCI 38 (2011) 3483-3496.

[7] Frankel David. and Webb Jennifer M: J Archaeol SCI 39 (2012) 1380-1387.

[8] L. Cheng, S.L. Feng, R.W. Li, W.J. Zhang, : J Radioan Nucl Ch 279(2) (2009) 681-683.

[9] H.M. Anawar, M.C. Freitas: J Radioan Nucl Ch 283 (2010)347-352.

[10] L.J. Mao, D.W. Mo, K. Hu, J.H. Yang: J Radioan Nucl Ch 285(2010) 359-364.

[11] L.J. Mao, D.W. Mo,, K. He, J.H. Yang, : Pedosphere 19(5) (2009) 615-622.

[12] L.J. Mao, D.W. Mo, J.H.Yang, Y.F. Jia, Y.Y. Guo: J Radioan Nucl Ch 295 (2013) 513-521.

[13] S.Y.Jiang, H.X. Zhao, Y. Q. Chen, T. Yang, J.H. Yang, H.F. Ling, : Chem Geol 244(2007) 584-604.

[14] D.J. Kennett, S. Sakai, H. Neff, R. Gossett, D.O. Larson: J Archaeol SCI 29 (2002) 443-455.

[15] S.R.Taylor, S.M. McLennan, The Continental Crust: Its Composition and Evolution. Blackwell, Oxford. 312. (1985).

[16] W.D. Kingery, P.B. Vandiver, Ceramic masterpieces, New York: Free Press. KD. (1986). 